\title{
ON THE EMBEDDING OF TOPOLOGICAL SEMIGROUPS AND INTEGRAL DOMAINS
}

\author{
B. GELBAUM, G. K. KALISCH, AND J. M. H. OLMSTED
}

1. Introduction. In the following the topological aspects of the classical theory of embedding semigroups and integral domains are considered. $\S 2$ is a summary of the relevant topological and algebraic concepts and theorems which are employed in the remainder of the paper. In $\$ 3$, the general theory is developed and applied to certain special cases. $\$ 4$ is devoted to the proof that a commutative semigroup with a cancellation law is a group if it is compact. In $\$ 5$ there is a discussion of metric semigroups and in $\$ 6$ a more extended treatment is given for complete separable metric semigroups.

2. Algebraic and topological preliminaries. A commutative semigroup with identity $e$ and cancellation law will be denoted by $S$. In the Cartesian product $S \times S$ let $R$ be the relation defined by the statement: $(a, b) R(c, d)$ if and only if $a d=b c$. The algebraic quotient structure $S \times S / R$ will be denoted by $Q(S)$. The canonical mapping of $S \times S$ onto $Q(S)$ will be denoted by $\phi$, and the set $\phi(S \times e)$ by $S^{\sharp}$. We shall designate integral domains by $I$ and their multiplicative semigroups by $I^{*} ; R, I \times I^{*} / R=Q(I), \phi$, and $I^{\#}$ will have analogous meanings.

A topological semigroup is a semigroup with a Hausdorff topology with respect to which multiplication is continuous in both variables. Topological groups and fields are the conventional entities. A topological integral domain is one whose additive group is topological and in which multiplication is continuous in both variables. In the case of a topological semigroup or integral domain, the relation $R$ will be called open in case, for any open set $U$ of $S \times S$ (of $I \times I^{*}$ ), the saturation of $U, \phi^{-1}(\phi(U))$, is open. We shall call $S(I)$ embeddable or weakly embeddable in $Q(S)(Q(I))$ if (a) $Q(S)(Q(I))$ is a topological group (field) and (b) $S^{\#}\left(I^{\#}\right)$ in the induced topology is a topological or continuous image of $S(I)$.

A topology on a given set will be called weaker than a second topology on the same set if every set that is open in the first topology is also open in the second. The second topology is then said to be stronger than the first. Thus weak embeddability of $S$ or $I$ means that the topology induced on $S^{\#}$ or $I^{\#}$ is weaker than the original.

Presented to the International Congress of Mathematicians, September 1, 1950; received by the editors July 20, 1950 and, in revised form, November 20, 1950. 
We observe that if $A$ is a topological space and if $\phi$ is a mapping of $A$ onto $B$, then there exists a unique strongest topology on $B$ with respect to which $\phi$ is continuous.

THEOREм 1. R-equivalence classes in $S \times S\left(I \times I^{*}\right)$ are closed sets in the product topology.

Proof. Let $A$ be an equivalence class and assume $(a, b)$ is in $\bar{A}$ (the closure of $A$ in $S \times S$ ). Let $U_{a}, U_{b}$ be any neighborhoods of $a$, $b$ in $S$. Then $\left(U_{a}, U_{b}\right)$ is a neighborhood of $(a, b)$ in $S \times S$ and consequently $\left(U_{a}, U_{b}\right)$ meets $A$. Fix $(c, d)$ in $A$. If it is false that $(a, b) R(c, d)$, then $a d \neq b c$, and hence there are neighborhoods $U_{a}$ and $U_{b}$ such that $d U_{a}$ and $c U_{b}$ do not meet. But then there is an element $\left(u_{a}, u_{b}\right)$ in $A$ and in $\left(U_{a}, U_{b}\right)$, and thus $u_{a} d=u_{b} c$. This is a contradiction. The proof for integral domains is identical.

We state the following theorem without proof.

THEOREM 2. If the relation $R$ is open and if $Q(S)(Q(I))$ is endowed with the strongest topology with respect to which $\phi$ is continuous, then the mapping $\phi$ is open, and conversely.

3. Topology of quotient structures. We shall now consider a criterion for the openness of the relation $R$. Since for any collection of sets in $I \times I^{*}$ the saturation of their union is the union of their saturations, the openness of $R$ is clearly equivalent to the openness of the saturations of sets of the form $\left(U_{a}, U_{b}\right)$. In fact, it is easily seen that openness is equivalent to the following: corresponding to any neighborhood of zero $U$ and equivalent pairs of points $(a, b)$ and $(c, d)$ in $I \times I^{*}$, there exists a neighborhood of zero $V$ such that every point of $(c+V, d+V)$ is equivalent to some point of $(a+U, b+U)$. A further simplification is possible. Because of the continuity of multiplication, if $a \in I$ and if $W$ is any open set in $I$, then the set $[x: a x \in W]$ is open. It follows trivially that if $x$ is a nonzero element of $I$ and if $(c, d)$ is any element of $I \times I^{*}$, then if $a=c x$ and $b=d x$, the conditions of the preceding criterion are satisfied, with $V$ $=[y: x y \in U]$. Since any two equivalent pairs, $(a, b)$ and $(c, d)$, are equivalent to a "common multiple," $(a d, b d)=(b c, b d)$, it is possible to formulate the following criterion.

THEOREM 3. The relation $R$ is open if and only if, corresponding to $a, b$, and $c$ in $I$, where $b \neq 0$ and $c \neq 0$, and a neighborhood of zero $U$, there exists a neighborhood of zero $V$ such that every point of $(a c+V$, $b c+V)$ is equivalent to some point of $(a+U, b+U)$.

For a commutative semigroup with cancellation law this criterion 
takes the form: The relation $R$ is open if and only if, corresponding to elements $a, b$, and $c$ and neighborhoods $U_{a}$ and $U_{b}$, there exist neighborhoods $U_{a c}$ and $U_{b c}$ such that every point of $\left(U_{a c}, U_{b c}\right)$ is equivalent to some point of $\left(U_{a}, U_{b}\right)$.

In case $I$ is a metric space (or one whose topology can be described by the convergence of simple sequences) the criterion for openness of $R$ can be stated as follows: The relation $R$ is open if and only if for arbitrary null sequences $a_{n}, b_{n}$ and arbitrary $a, b(\neq 0), c(\neq 0)$ one can find null sequences $d_{n}, e_{n}$ with the property $\left(a+d_{n}, b+e_{n}\right) R\left(c a+a_{n}\right.$, $\left.c b+b_{n}\right)$. A similar observation applies to $S$.

THEOREM 4. If $R$ is open and if $Q(S)(Q(I))$ is endowed with the strongest topology with respect to which $\phi$ is continuous, then $Q(S)$ $(Q(I))$ is a topological group (field).

Proof. We prove only that addition in $Q(I)$ is continuous. Proofs for all other operations and cases are similar. Thus let $r, s$ belong to $Q(I), r=\phi(a, b), s=\phi(c, d), r+s=t$. If $U_{t}$ is a neighborhood of $t$ and if $\phi(x, y)=t$, then one can find neighborhoods $U_{x}, U_{y}$ such that $\phi\left(U_{x}, U_{y}\right) \subset U_{t}$. Since $\phi(a d+b c, b d)=t$, there are neighborhoods $U_{a}, U_{b}, U_{c}, U_{d}$ for which $\phi\left(U_{a} U_{d}+U_{b} U_{c}, U_{b} U_{d}\right) \subset U_{t}$. It follows that if we set $U_{r}=\phi\left(U_{a}, U_{b}\right), U_{s}=\phi\left(U_{c}, U_{d}\right)$, then $U_{r}+U_{s} \subset U_{t}$, and the sets $U_{r}, U_{s}$ are open since $\phi$ is open. The openness of the mapping $\phi$, the continuity of multiplication in $I$, and the fact that $I$ is a Hausdorff space imply that $Q(I)$, as topologized, is also a Hausdorff space. (Alternately, recalling Theorem 1 and the continuity of $\phi$, we see that $Q(I)$, as topologized, is a $T_{1}$-space. Since it is also a field with continuous operations, it follows that $Q(I)$ is a Hausdorff space.)

An example of a topological integral domain that is embeddable in a topological field, but for which the relation $R$ is not open: Let $I$ be the polynomials whose coefficient field is the rational numbers with a $p$-adic valuation. The valuation for a polynomial is the maximum of the valuations of the coefficients. The quotient field has a valuation given by quotients of valuations of polynomials. The polynomials are not dense in the rational functions (the function $1 / x$ is at a distance of at least one from any polynomial). Therefore, by Theorem 12 , below, the relation $R$ is not open.

Theorem 5. If $S(I)$ is embeddable in $Q(S)(Q(I))$, then $\phi$ is continuous on $S \times S\left(I \times I^{*}\right)$.

Proof. Consider the case of $S$. The proof for $I$ is identical. Let $x=\phi(a, b)$ belong to $Q(S)$. Then for a given neighborhood of $x, U_{x}^{\prime}$, there are neighborhoods $U_{a}^{\prime}, U_{b}^{\prime}$ of $a$ and $b$ in $Q(S)$ such that 
$U_{a}^{\prime}\left(U_{b}^{\prime}\right)^{-1} \subset U_{x}^{\prime}$. (Here we have identified $a$ and $b$ with $\phi(a, e)$ and $\phi(b, e)$.) Let $U_{a}=U_{a}^{\prime} \cap S^{\prime}, U_{b}=U_{b}^{\prime} \cap S^{f}$. These are open sets in $S^{\prime}$ and $\phi\left(U_{a}, U_{b}\right) \subset U_{x}^{\prime}$, whence $\phi$ is continuous.

THEOREM 6. If $I$ is weakly embeddable in $Q(I)$ in the strongest topology relative to which $\phi$ is continuous and if $Q(I)$ in some other topology is a topological field $F$ in which $I$ is embedded, then $I$ is embeddable in $Q(I)$ (in its original topology). A similar result holds for semigroups.

Proof. By Theorem 5, $\phi$ is continuous relative to the topology of $F$, and thus the topology of $Q(I)$ is stronger than that of $F$. Consequently the topology induced on $I^{*}$ by $Q(I)$ is at least as strong as that induced on $I^{H}$ by $F$. The latter topology is the topology of $I$, and thus the result follows, since in the former topology we have at most a weakening.

THEOREM 7. Let $F$ be a topological field, I and $I^{\prime}$ topological integral domains, and assume that $I \subset I^{\prime} \subset F$, where the inclusions are algebraic and topological. If $F=Q(I)$, where $Q(I)$ has been provided with the strongest topology with respect to which the mapping $\phi$ is continuous, then $F=Q\left(I^{\prime}\right)$, where $Q\left(I^{\prime}\right)$ has been provided with the strongest topology with respect to which $\phi^{\prime}$ (the mapping of $I^{\prime} \times I^{\prime *}$ on $Q\left(I^{\prime}\right)$ ) is continuous. $A$ similar result holds for semigroups and groups.

Proof. Clearly $F$ is the algebraic quotient structure of $I^{\prime}$. Furthermore since $I^{\prime}$ is topologically embedded in $F, \phi^{\prime}$ is continuous (Theorem 5). We show that the topology of $F$ is the strongest relative to which $\phi^{\prime}$ is continuous. Otherwise, $\phi$ (which is the contraction of $\phi^{\prime}$ to $I \times I^{*}$ ) would be continuous relative to a topology definitely stronger than the strongest with respect to which it is continuous, a contradiction. The proof for the case of semigroups and groups is similar.

THEOREM 8. If $S(I)$ has the property that $U$ open implies a $U$ open ( $a \neq 0$ in the case of an integral domain), then the relation $R$ is open.

PRoof. By use of Theorem 3 we have only to let $U_{a c}=c U_{a}$ and $U_{b c}=c U_{b}(V=c U)$.

THEOREM 9. If $S(I)$ is algebraically a group (field) and topologically a semigroup (integral domain), the relation $R$ is open and the topology can be weakened so that $S(I)$ becomes a topological group (field).

Proof. For the case $I:$ If $a \neq 0$ and if $U$ is open, then $a U$ is the inverse image of the open set $U$ under the continuous transformation $x \rightarrow a^{-1} x$, and is therefore open. By Theorem 8 , the relation $R$ is open. 
Therefore the quotient field can be given a topology that makes it a topological field and that induces upon $I$ a possibly weaker topology than the original. But $I$ is algebraically identical with its quotient field. A similar proof holds for $S$.

CoRollary. If in the above $S$ is compact, then $S$ is a topological group.

THEOREM 10. If $S(I)$ is a topological group (field), it is identical with $Q(S)(Q(I))$, where the topology of the latter is the strongest relative to which $\phi$ is continuous.

Proof. Only the proof for $I$ will be given. By Theorem $9, R$ is open, whence $Q(I)$ in the strongest topology relative to which $\phi$ is continuous is a topological field in which $I$ is weakly embedded. Since in the original topology of $I$ (which is $Q(I)$ ) $I$ is actually embedded, Theorem 6 shows that $I$ is embedded in $Q(I)$ in the first topology mentioned.

THEOREM 11. If $I$ is embedded densely in a topological field $F$, the relation $R$ is open.

Proof. Let $U_{I}$ be any neighborhood of zero, in $I$, and let $U_{F}$ be an open set in $F$ whose intersection with $I$ is $U_{I}$. Let $a, b, c \in I, b \neq 0$, $c \neq 0$. Let $V_{F}$ be a neighborhood of zero in $F$ such that, for any elements $v_{i}$ of $V_{F},(1 / c) v_{1}+a c v_{2}+v_{1} v_{2}$ and $(1 / c) v_{3}+b c v_{4}+v_{3} v_{4} \in U_{F}$. Let $V_{I}=V_{F} \cap I$. If $(x, y)$ is any element of $\left(a c+V_{I}, b c+V_{I}\right)$ and if $z$ is any element of $I \cap\left((1 / c)+V_{F}\right)$ (the intersection is not empty), then $(x z, y z)$ has the form $\left(\left(a c+v_{1}\right)\left((1 / c)+v_{2}\right), \quad\left(b c+v_{3}\right)\left((1 / c)+v_{2}\right)\right)$ $=\left(a+(1 / c) v_{1}+a c v_{2}+v_{1} v_{2}, \quad b+(1 / c) v_{3}+b c v_{2}+v_{3} v_{2}\right)=\left(a+u_{1}, \quad b+u_{2}\right)$, where $u_{1}$ and $u_{2} \in U_{F}$. Since $x z, y z, a$, and $b \in I, u_{1}$ and $u_{2} \in U_{I}$. Theorem 3 completes the proof.

THEOREM 12. If I is a polynomial integral domain with a nondiscrete coefficient field (whose topology is induced by that of I), topologically embedded in its quotient field $F=Q(I)$ (with a topology relative to which $F$ is a topological field), and if the relation $R$ is open, then $I$ is dense in $F$.

Proof. Let $c$ be a nonzero polynomial in $I$ and let $U_{F}$ be a neighborhood of zero in $F$. Let $V_{F}$ be a neighborhood of zero in $F$ such that whenever $x$ is in $1+V_{F}$ and $z$ is in $V_{F}$ then $x /(c+z)$ is in $(1 / c)+U_{F}$. Because of the openness of the relation and the fact that $(c, c) R(1,1)$, corresponding to $V_{I}=I \cap V_{F}$, there exists a neighborhood of zero $W_{I} \subset V_{I}$ such that if $w_{1}$ and $w_{2} \in W_{I}$, there exist elements $v_{1}$ and $v_{2}$ of $V_{I}$ such that $\left(c+w_{1}, c+w_{2}\right) R\left(1+v_{1}, 1+v_{2}\right)$. Let $\lambda$ be a nonzero constant such that $\lambda$ and $2 \lambda \in W_{I}$. Then there exist $x$ and $y \in 1+V_{I}$ 
such that $(c+\lambda, c+2 \lambda) R(x, y)$. Therefore $(c+2 \lambda) x=(c+\lambda) y$, and since $c+\lambda$ and $c+2 \lambda$ are relatively prime (their difference is $\lambda$ ), there exists a polynomial $k$ such that $x=k(c+\lambda), y=k(c+2 \lambda)$. In $F$, $k=x /(c+\lambda)$. But $x \in 1+V_{I}$ and $\lambda \in W_{I} \subset V_{I}$. Therefore $k \in(1 / c)+U_{F}$.

THEOREM 13. Let $I_{p}$ be the integers in their $p$-adic topology ( $p$ a prime), and assume that $Q\left(I_{p}\right)$ is provided with the strongest topology with respect to which $\phi$ is continuous. Then (a) $\phi$ is open, (b) $Q\left(I_{p}\right)$ is a topological field, (c) $R_{p}$ (the rationals in their p-adic topology) and $Q\left(I_{p}\right)$ are identical (algebraically and topologically).

Proof. By one of the alternative formulations of the criterion of Theorem 3 , since $I_{p} \times I_{p}^{*}$ satisfies the first countability axiom, it suffices to show that if $a_{n}, b_{n}$ are null sequences in $I_{p}$ and if $c, f$, and $g$ are arbitrary in $I_{p}$ and $c g \neq 0$, one can find null sequences $d_{n}, e_{n}$ such that $\left(f+d_{n}, g+e_{n}\right) R\left(c f+a_{n}, c g+b_{n}\right)$. This means that

$$
e_{n}\left(c f+a_{n}\right)-d_{n}\left(c g+b_{n}\right)=f b_{n}-g a_{n} \equiv h_{n} .
$$

Note that if $r$ divides $c f+a_{n}$ and $c g+b_{n}$, then $r$ divides $f b_{n}-g a_{n}$ since $f b_{n}-g a_{n}=f\left(c g+b_{n}\right)-g\left(c f+a_{n}\right)$. Let $A_{n}=c f+a_{n}, B_{n}=c g+b_{n}, s_{n}=$ the greatest common divisor of $A_{n}$ and $B_{n}$. There is an integer $N$ independent of $n$ such that for $m>N, p^{m} \nmid A_{n}, p^{m} \nmid B_{n}$. Thus if we set $P_{n}$ $=A_{n} / s_{n}, Q_{n}=B_{n} / s_{n}$, there exist integers $R_{n}, S_{n}$ for which $R_{n} P_{n}-S_{n} Q_{n}$ $=1$, and $h_{n} R_{n} P_{n}-h_{n} S_{n} Q_{n}=h_{n}$. Since $a_{n}, b_{n}$ are null sequences, $h_{n}$ approaches 0 and hence $h_{n}$ contains a factor $p^{t_{n}}$, with $t_{n}$ approaching infinity. Let $s_{n}=k_{n} p^{u_{n}}$, where $k_{n}$ is prime to $p$. Then the numbers $u_{n}$ are bounded and consequently $h_{n} / s_{n}=\phi\left(h_{n}, s_{n}\right)$ approaches 0 , since $\phi$ is continuous and $h_{n}$ approaches 0 . Let $d_{n}=h_{n} R_{n} / s_{n}, e_{n}$ $=h_{n} S_{n} / s_{n}$. Then $d_{n}, e_{n}$ are integers, since $h_{n} / s_{n}$ are integers; since the $u_{n}$ are bounded, it follows that $d_{n}$ and $e_{n}$ are null sequences, and the above computations show that they fulfill the requirements which were assigned. Thus $\phi$ is open, and part (a) is proved.

(b) By Theorem 2, $Q\left(I_{p}\right)$ is a topological field.

(c) Since $I_{p}$ is embeddable in $R_{p}, \phi$ relative to $R_{p}$ is continuous by Theorem 5. Thus the topology of $R_{p}$ is comparable with and not strictly stronger than the topology of $Q\left(I_{p}\right)$. Since $\phi$ relative to $Q\left(I_{p}\right)$ is open and since $I_{p} \times I_{p}^{*}$ satisfies the first countability axiom, so does $Q\left(I_{p}\right)$. Thus to show that the topology of $R_{p}$ is the same as that for $Q\left(I_{p}\right)$ we need only show that if $r_{n}$ approaches $r$ in $R_{p}$, then $r_{n}$ approaches $r$ in $Q\left(I_{p}\right)$. Since both $R_{p}$ and $Q\left(I_{p}\right)$ are topological fields, we may assume $r=0$. Thus $r_{n}=\left(k_{n} / m_{n}\right) p^{t_{n}}$, where the $t_{n}$ approach infinity and $k_{n}$ and $m_{n}$ are relatively prime, and prime to $p$. Then $\phi\left(k_{n} p^{t_{n}}, m_{n}\right)=r_{n}$ and there are integers $a_{n}$ and $b_{n}$ such that $a_{n} m_{n}$ 
$+b_{n} p^{n}=1$. Thus $r_{n}=\phi\left(a_{n} k_{n} p^{t_{n}}, a_{n} m_{n}\right)=\phi\left(a_{n} k_{n} p^{t_{n}}, 1-b_{n} p^{n}\right)$. Clearly $a_{n} k_{n} p^{t_{n}}$ is a null sequence, and $1-b_{n} p^{n}$ approaches 1 . Thus $r_{n}$ approaches $\phi(0,1)=0$ in $Q\left(I_{p}\right)$, since $\phi$ is continuous relative to the topology of $Q\left(I_{p}\right)$.

COROLLARY. If $I$ is any integral domain algebraically and topologically contained in $R_{p}, Q(I)$ is $R_{p}$ when $Q(I)$ is provided with the strongest topology with respect to which $\phi$ is continuous, and I is embeddable in $Q(I)$.

PROOF. This is a consequence of Theorem 7.

4. Compact semigroups. In this section we temporarily abandon the conventions of notation established above.

THEOREM 14. Let $S$ be a semigroup with a two-sided cancellation law (we do not assume commutativity or the existence of an identity). If $S$ is a compact Hausdorff space and if multiplication is continuous in both variables, then $S$ is a topological group in its given topology. ${ }^{1}$

Proof. We shall need two lemmas for the proof of the above. The first is a mere exercise in algebra and its proof will not be given.

Lemma 1. If $S$ is a semigroup with a two-sided cancellation law (no commutativity or existence of identity assumed) and if there are two elements $a, b$ in $S$ which satisfy $b=a b$, then $S$ has a unique (two-sided) identity.

Lemma 2. If $G$ is a group and a compact Hausdorff space and if multiplication is continuous in both variables, then $G$ is a topological group, that is, $x^{-1}$ is a continuous function of $x$.

Remarks. In Theorem 14 and in Lemma 2, compactness is to be construed in the sense that every infinite set of $S$ or of $G$ has a limit point (that is, $S$ and $G$ are countably compact). As is well known, spaces compact in the Bourbaki-Tukey sense (Alexandroff-Urysohn: "bicompact") and sequentially compact spaces are countably compact. In what follows we shall use the notations of [5].2

Proof. Assume $x(\delta) \rightarrow_{\Delta} x_{0}$. Then $(x(\delta))^{-1}$ is a set of points having a limit point $y$, and thus there is a function $\alpha(\delta)$ which is cofinal in $\Delta$ and such that $(x(\alpha(\delta)))^{-1} \rightarrow_{\Delta} y$. Continuity of multiplication now implies that $y=x_{0}^{-1}$. A similar argument shows that there is only one limit point of the points $(x(\delta))^{-1}$ and the continuity of division follows.

${ }^{1}$ See discussion following the proof of Theorem 14.

2 Numbers in brackets refer to the bibliography at the end of the paper. 
Proof OF THE THEOREM. Let $x$ be an arbitrary element of $S$. In view of Lemma 1 , we may assume that the elements $x^{n}, n=1,2, \cdots$, are distinct. Thus they have a limit point $z_{0}$, and there is a function $n(\delta)$ such that $x^{n(\delta)} \rightarrow \Delta z_{0}$. Clearly we may assume that all $n(\delta)$ are greater than 1 . Thus the points $x^{n(\delta)-1}$ have a limit point $z_{1}$, and there is a function $\alpha(\delta)$ cofinal in $\Delta$ for which $x^{n(\alpha(\delta))-1} \rightarrow \Delta z_{1}$, whence $x z_{1}=z_{0}$, owing to the continuity of multiplication. In this manner one constructs a sequence $z_{n}$ such that $x^{n} z_{n}=z_{0}$ (the $z_{n}$ will be distinct!). There is a function $\beta(\delta)$ which is cofinal in $\Delta$ and for which $z_{n(\beta(\delta))} \rightarrow \Delta z^{\prime}$, where $z^{\prime}$ is a limit point of the sequence $z_{n}$. Hence $x^{n(\beta(\delta))} z_{n(\beta(\delta))} \rightarrow \Delta z_{0} z^{\prime}$ $=z_{0}$, and we conclude, using Lemma 1 , that $S$ has an identity.

Since $x S$ is again a semigroup satisfying the hypotheses of Theorem 14 , it follows that $x S$ has an identity which is easily seen to be the identity of $S$. Thus $x^{-1}$ exists and $S$ is a group. An application of Lemma 2 yields the desired result.

Note that the Hausdorff property of the space is employed only to insure the uniqueness of limits. If we relax this restriction and demand only that points be closed sets $\left(T_{1}\right)$, the theorem fails to hold as the following example shows: Let $N$ be the non-negative natural numbers, clearly an additive (indeed abelian) semigroup with a cancellation law. Topologize $N$ by defining the neighborhoods of a point $n$ as follows: a neighborhood of $n$ consists of the point $n$ and the complement of some finite set of integers. Thus topologized, $N$ is a compact $T_{1}$ space which is not a Hausdorff space, and addition is continuous in both variables.

Iwasawa has published a proof of Theorem 14 in Sūgaku vol. 1 (1947), for the case of $S$ bicompact. At the suggestion of the editors we include a brief sketch of his proof: By Zorn's lemma there is a minimal nonvacuous closed subset $A$ of $S$ such that $A S \subset A$. Thus for $a$ in $A, a S=A$. If $x$ is in $S, a$ in $A$, then $a x$ is in $A, a x S=A=a S$, and $x S=S$. Dually, $S x=S$, whence $S$ is a group. The class of all left and right translations of $S$ is equicontinuous since multiplication is continuous on the compact space $S \times S$ and therefore uniformly continuous on $S \times S$. The continuity of inversion is shown as follows: if $x$ is close to $y$, then $x y^{-1}$ is close to the identity and $y^{-1}$ is close to $x^{-1}$. According to a personal communication, J. E. L. Peck has obtained a similar result.

5. Metric semigroups. If $S$ is a semigroup with an invariant metric, then $S$ is a topological semigroup since $a_{n} \rightarrow a, b_{n} \rightarrow b$ imply $d\left(a_{n} b_{n}, a b\right)$ $\leqq d\left(a_{n} b_{n}, a_{n} b\right)+d\left(a_{n} b, a b\right)=d\left(b_{n}, b\right)+d\left(a_{n}, a\right) \rightarrow 0, a_{n} b_{n} \rightarrow a b$, where $d$ is the distance in $S$. We now introduce a norm in $Q(S)$ as follows: if $A=\phi(a, b)$ is in $Q(S)$, we define 


$$
\|A\|=\inf _{\left(a^{\prime}, b^{\prime}\right) R(a, b)}\left(d\left(a^{\prime}, e\right)+d\left(b^{\prime}, e\right)\right) .
$$

Regarding this norm the following theorem holds.

THEOREM 15. Let $S$ be as above and let $Q(S)$ be metrized by $D(A, B)$ $=\left\|A B^{-1}\right\|$. Then $Q(S)$ is a topological group with an invariant metric $D ; Q(S)$ is discrete if and only if $S$ is discrete; $\phi$ is open on $S \times S ; \phi$ restricted to $S \times e$ is open and continuous at $e$; finally, if $R$ is open, $\phi$ restricted to $S \times e$ is continuous and thus $S$ is embeddable in a topological group with an invariant metric.

Proof. 1. $\|\cdot\|$ is a norm of $Q(S)$ : Clearly $\|A\| \geqq 0$ and $A=E$, the identity of $Q(S)$, implies $\|A\|=0$. If $\|A\|=\|\phi(a, b)\|=0$, there exist sequences $a_{n}, b_{n}$ of elements of $S$ such that $\left(a_{n}, b_{n}\right) R(a, b), a_{n} \rightarrow e$, $b_{n} \rightarrow e$, whence, since $a_{n} b=a b_{n}, b=a$ and $A=E$. We next observe that $\|A B\| \leqq\|A\|+\|B\|$. For let $A=\phi\left(a_{1}, a_{2}\right)$ and $B=\phi\left(b_{1}, b_{2}\right), A B$ $=\phi\left(a_{1} b_{1}, a_{2} b_{2}\right)$. Given any positive $\epsilon$, select $a_{1}^{\prime}, b_{1}^{\prime}, a_{2}^{\prime}, b_{2}^{\prime}$ such that $\left(a_{1}^{\prime}, a_{2}^{\prime}\right) R\left(a_{1}, a_{2}\right),\left(b_{1}^{\prime}, b_{2}^{\prime}\right) R\left(b_{1}, b_{2}\right)$ and $d\left(a_{1}^{\prime}, e\right)+d\left(a_{2}^{\prime}, e\right) \leqq\|A\|+\epsilon$, $d\left(b_{1}^{\prime}, e\right)+d\left(b_{2}^{\prime}, e\right) \leqq\|B\|+\epsilon$. Now $\|A B\| \leqq d\left(a_{1}^{\prime} b_{1}^{\prime}, e\right)+d\left(a_{2}^{\prime} b_{2}^{\prime}, e\right)$ since $\left(a_{1}^{\prime} b_{1}^{\prime}, a_{2}^{\prime} b_{2}^{\prime}\right) R\left(a_{1} b_{1}, a_{2} b_{2}\right)$. But $d\left(a_{1}^{\prime} b_{1}^{\prime}, e\right)+d\left(a_{2}^{\prime} b_{2}^{\prime}, e\right) \leqq d\left(a_{1}^{\prime} b_{1}^{\prime}, b_{1}^{\prime}\right)$ $+d\left(b_{1}^{\prime}, e\right)+d\left(a_{2}^{\prime} b_{2}^{\prime}, \quad b_{2}^{\prime}\right)+d\left(b_{2}^{\prime}, e\right)=d\left(a_{1}^{\prime}, e\right)+d\left(b_{1}^{\prime}, e\right)+d\left(a_{2}^{\prime}, e\right)$ $+d\left(b_{2}^{\prime}, e\right) \leqq\|A\|+\|B\|+2 \epsilon$ and $\|A B\| \leqq\|A\|+\|B\|$ as promised. Note further that $\|A\|=\left\|A^{-1}\right\|:\|A\|=\|\phi(a, b)\|=\inf _{\left(a^{\prime}, b^{\prime}\right) R(a, b)}\left(d\left(a^{\prime}, e\right)\right.$ $\left.+d\left(b^{\prime}, e\right)\right)=\inf _{\left(b^{\prime}, a^{\prime}\right) R(b, a)}\left(d\left(b^{\prime}, e\right)+d\left(a^{\prime}, e\right)\right)=\|\phi(b, a)\|=\left\|A^{-1}\right\|$. If now we define $D(A, B)=\left\|A B^{-1}\right\|$, we see that $D$ is an invariant metric of $Q(S)$; the continuity of multiplication and division is a consequence of the invariance of the metric.

2. $\phi$ restricted to $S \times e$ is continuous at the identity: if $A=\phi(a, e)$, $D(A, E)=\|A\|=\inf _{\left(a^{\prime}, b^{\prime}\right) R(a, e)}\left(d\left(a^{\prime}, e\right)+d\left(b^{\prime}, e\right)\right) \leqq d(a, e)$. Therefore, $a_{n} \rightarrow e$ implies that $A_{n} \rightarrow E$.

3. $\phi$ is open. Let $A_{n}=\phi\left(a_{n}, b_{n}\right) \rightarrow \phi(a, b)=A$. We wish to prove that there exist sequences $a_{n}^{\prime}, b_{n}^{\prime}$ of elements of $S$ such that $\left(a_{n}^{\prime}, b_{n}^{\prime}\right) R\left(a_{n}, b_{n}\right)$ and $\left(a_{n}^{\prime}, b_{n}^{\prime}\right) \rightarrow(a, b)$. Now $\inf _{\left(a^{\prime}, b^{\prime}\right) R\left(a_{n} b, a b_{n}\right)}\left(d\left(a^{\prime}, e\right)\right.$ $\left.+d\left(b^{\prime}, e\right)\right) \rightarrow 0$ implies that there exist sequences $a_{n}^{\prime \prime}, b_{n}^{\prime \prime}$ of elements of $S$ such that $d\left(a_{n}^{\prime \prime}, e\right) \rightarrow 0, d\left(b_{n}^{\prime \prime}, e\right) \rightarrow 0,\left(a_{n}^{\prime \prime}, b_{n}^{\prime \prime}\right) R\left(a_{n} b, a b_{n}\right)$ or $a_{n}^{\prime \prime} a b_{n}$ $=a_{n} b b_{n}^{\prime \prime}$; if we let $a_{n}^{\prime}=a_{n}^{\prime \prime} a, b_{n}^{\prime}=b_{n}^{\prime \prime} b$, our assertion is proved.

4. $\phi$ restricted to $S \times e$ is open. Let $A_{n}=\phi\left(a_{n}, e\right) \rightarrow \phi(a, e)$ $=A$; to show that $a_{n} \rightarrow a$. We see that $\left\|A_{n} A^{-1}\right\|=\left\|\phi\left(a_{n}, a\right)\right\|$ $=\inf _{\left(a^{\prime}, b^{\prime}\right) R\left(a_{n}, a\right)}\left(d\left(a^{\prime}, e\right)+d\left(b^{\prime}, e\right)\right) \rightarrow 0$. Hence, as above, there exist sequences $a_{n}^{\prime}, b_{n}^{\prime}$ of elements of $S$ such that $a_{n}^{\prime} \rightarrow e, b_{n}^{\prime} \rightarrow e, a_{n}^{\prime} a=a_{n} b_{n}^{\prime}$. Finally $d\left(a_{n}, a\right)=d\left(a_{n} b_{n}^{\prime}, a b_{n}^{\prime}\right)=d\left(a_{n}^{\prime} a, a b_{n}^{\prime}\right)=d\left(a_{n}^{\prime}, b_{n}^{\prime}\right) \leqq d\left(a_{n}^{\prime}, e\right)$ $+d\left(e, b_{n}^{\prime}\right) \rightarrow 0$, and our assertion is proved. The fact that $Q(S)$ is discrete if and only if $S$ is discrete is a consequence of 2 and 4. 
5. Let now $R$ be open; we wish to show that $\phi$ contracted to $S \times e$ is continuous, or that $a_{n} \rightarrow a$ implies that $A_{n}=\phi\left(a_{n}, e\right) \rightarrow A=\phi(a, e)$. But $D\left(A_{n}, A\right)=\left\|A_{n} A^{-1}\right\|=\inf _{\left(a^{\prime}, b^{\prime}\right) R\left(a_{n}, a\right)}\left(d\left(a^{\prime}, e\right)+d\left(b^{\prime}, e\right)\right)$. Since $\left(a_{n}, a\right) \rightarrow(a, a)$ and $(a, a) R(e, e)$, we have the existence of sequences $a_{n}^{\prime}, b_{n}^{\prime}$ of elements of $S$ such that $\left(a_{n}^{\prime}, b_{n}^{\prime}\right) R\left(a_{n}, a\right),\left(a_{n}^{\prime}, b_{n}^{\prime}\right) \rightarrow(e, e)$ because $R$ is open and $S \times S$ and $Q(S)$ are metric (see paragraph immediately preceding Theorem 4$)$. This last relation shows that $D\left(A_{n}, A\right)=\inf _{\left(a^{\prime}, b^{\prime}\right) R\left(a_{n}, a\right)}\left(d\left(a^{\prime}, e\right)+d\left(b^{\prime}, e\right)\right) \leqq d\left(a_{n}^{\prime}, e\right)+d\left(b_{n}^{\prime}, e\right) \rightarrow 0$ as promised.

ThEOREM 16. Let $S$ be a metric topological semigroup such that $R$ is open. Then the property

$$
a_{n} b_{n} \rightarrow b, a_{n} \rightarrow e \text { implies } b_{n} \rightarrow b
$$

is a necessary and sufficient condition for embeddability.

Proof. The necessity is obvious. The sufficiency is proved as follows. In the strongest topology on $Q(S)$ relative to which $\phi$ is continuous, $\phi$ is an open continuous mapping by Theorem 2. $\phi$ contracted to $S \times e$ is continuous. Let now $y_{n} \rightarrow y$, where $y_{n}$ and $y$ are in $S^{\prime}$, $y_{n}=\phi\left(x_{n}, e\right)$ and $y=\phi(x, e)$. Since $\phi$ is open there exist sequences $b_{n}, c_{n}$ of elements of $S$ such that $y_{n}=\phi\left(b_{n}, c_{n}\right),\left(b_{n}, c_{n}\right) \rightarrow(x, e)$; hence $\left(b_{n}, c_{n}\right) R\left(x_{n}, e\right)$ or $b_{n}=c_{n} x_{n}$ where $c_{n} \rightarrow e, b_{n} \rightarrow x$, hence, by (1), $x_{n} \rightarrow x ; \phi$ contracted to $S \times e$ is open, whence our assertion.

Note that since an invariant metric implies (1), the last part of Theorem 15 is a consequence of the preceding theorem. We also note the following consequences of the foregoing: if $S$ as described in our theorem is (algebraically) a group, it is a topological group. For future reference we observe that the following is a necessary and sufficient condition for embeddability which is a stronger necessary condition than (1): if $a_{n} b_{n} \rightarrow c$, and $a_{n} \rightarrow a$, then $b_{n}$ converges.

6. Complete separable metric semigroups. Except when the contrary is indicated all semigroups $S$ mentioned hereinafter are complete separable metric topological semigroups with the additional property:

(2) If $U$ is an open subset of $S$, then so is aU for all $a$ in $S$ (cf. Theorem 8).

LemMa 3. Let $\{U\}$ be a basis of neighborhoods of e. Then $\{a U\}$ is a basis of neighborhoods of a. If $f$ is a homomorphism of $S$ onto $S^{\prime}=f(S)$ which is continuous at $e$, then it is continuous.

Proof. This lemma uses only property (2) and the continuity of $a b$ in each variable. 1 . Since $a U$ is open and since every open set contain- 
ing $a$ contains some $a U$ (by one-sided continuity of $a b$ ), the first assertion is clear. 2. Let now $U^{\prime}$ be a neighborhood of $e^{\prime}=f(e)$. There exists a neighborhood $V$ of $e$ such that $f(V)=V^{\prime}$ is contained in $U^{\prime}$. Consider now $a^{\prime}$ in $S^{\prime}$ and $a^{\prime} U^{\prime}$; since $V^{\prime}$ is in $U^{\prime}, a^{\prime} V^{\prime}=(a V)^{\prime}$ is in $a^{\prime} U^{\prime}$ which proves the second assertion.

Note. If $S$ is any topological semigroup and $\{U\}$ a basis of neighborhoods of $e$, then if we retopologize $S$ by letting $\{a U\}$ be a basis at $a$, we get a stronger topology of $S$ with respect to which $S$ is still a topological semigroup. For, by continuity of multiplication, every open set containing $a$ contains some $a U$, and for each $a u$ in $a U$ there exists $U_{1}$ such that $a u U_{1}$ is in $a U$ : for $u$ in $U$ there exists $U_{1}$ such that $u U_{1}$ is in $U$ and, therefore, $a u U_{1}$ is in $a U$. The new topology of $S$ has at least as strong separation properties $\left(T_{0}, T_{1}, T_{2}\right)$ as the old one, and, of course, satisfies condition (2) above. Now a semigroup with property (2) automatically has one-sided continuity of multiplication: $a(b U)$ is in $(a b) U$; and the simultaneous continuity of multiplication in $S$ in the stronger topology is a consequence of the same property at $e$ in the original topology: given $U$ there exists $U_{1}$ such that $U_{1}^{2}$ is in $U$; hence $a U_{1} b U_{1}=a b U_{1}^{2}$ is in $a b U$.

Lemma 4. If $C_{i}$ is a subset of $S$ of the ith category $(i=1,2)$, then so is $a C_{i}$ for all $a$ in $S$; and conversely, if $a C$ is of the ith category, then so is $C$.

Proof. 1. Let the subset $N$ of $S$ be nowhere dense in $S$; we prove that $a N$ is nowhere dense in $S$. We first observe that $S$ and $a S$ are homeomorphic, because $S \rightarrow a S$ is open by hypothesis, continuous by one-sided continuity of multiplication, and 1-1 by the cancellation law. (Note, incidentally, that the homeomorphism of $S$ and $a S$, although implied by condition (2), is weaker than (2), as is shown by the example of the additive semigroup of the nonnegative reals with the usual topology.) We observe that if $(a X)^{c c}$ denotes the relative closure of $a X$ in $a S$, then $(a X)^{c c}=a X^{c}$ (where the single superscript $c$ denotes the closure in $S):(a X)^{c c}=(a X)^{c} \cap a S$ since $y$ in $(a X)^{c c}$ is equivalent to $y^{\prime}$ s being in $(a X)^{c}$ and in $a S$. Now, $(a X)^{c} \cap a S=a X^{c}$. We know by continuity of multiplication that $a X^{c}$ is a subset of $(a X)^{c}$; hence $a X^{c} \cap a S=a X^{c}$ is in $(a X)^{c} \cap a S$. Let now $z=a y$ be in $(a X)^{c} \cap a S$. Since $a y U$ is open, where $U$ is a neighborhood of $e$, ay $U \cap a X$ is not empty; therefore, $y U \cap X$ is not empty, since $\{y U\}$ is a basis of neighborhoods of $y$ by the first part of Lemma 3. We can conclude that $y$ is in $X^{c}, z=a y$ is in $a X^{c}$ and, therefore, $(a X)^{c} \cap a S$ is a subset of $a X^{c},(a X)^{c c}=(a X)^{c} \cap a S=a X^{c}$. Finally, in order to prove that $a N$ is nowhere dense, assume, on the contrary, that there 
exists an open set $G$ such that $G$ is dense in $(a N)^{c}$. Then $G \cap a S$ is not empty since $g$ in $G$ implies that $g$ is in $(a N)^{c}$; therefore, $G \cap a N$ is not empty, and $G \cap a S$ is not empty. Now $G \cap a S$ is a subset of $(a N)^{c} \cap a S=a N^{c}$. But since $N$ is nowhere dense in $S, a N$ is nowhere dense in $a S$ (by 1 above); hence $(a N)^{c c}$ contains no set open in $a S$; but $(a N)^{c c}=a N^{c}$ and $G \cap a S$ is open in $a S$, a contradiction. We now can conclude that $a C_{1}$ is of the first category in $S$ if $C_{1}$ is of the first category in $S$.

2. Let $a N$ be nowhere dense in $S$; we wish to prove that $N$ is nowhere dense in $S$. Assume, on the contrary, that there exists an open set $G$ in $N^{c}$; then the open set $a G$ is in $a N^{c}$ which in turn is contained in $(a N)^{c}$ and $a N$ is not nowhere dense, a contradiction. Again we can conclude that if $a C$ is of the first category in $S$, so is $C$.

3. The statements concerning $C_{2}, a C_{2}$, and the converse follow.

Lemma 5. Let the semigroup $S$ have the property

$$
\text { if } a_{n} b_{n} \rightarrow e, b_{n} \rightarrow e \text {, then } a_{n} \rightarrow e .
$$

Then, if $x_{n} y_{n} \rightarrow y$ and $y_{n} \rightarrow y, x_{n} \rightarrow e$.

Proof. Since $y U$ is open for open $U, x_{n} y_{n}$ and $y_{n}$ are in $y U$ for sufficiently large $n$; therefore, $x_{n} y_{n}=y e_{n}, y_{n}=y e_{n}^{\prime}$ where $e_{n} \rightarrow e, e_{n}^{\prime} \rightarrow e$; hence $x_{n} y_{n}=x_{n} y e_{n}^{\prime}=y e_{n}, x_{n} e_{n}^{\prime}=e_{n}$ and $x_{n} \rightarrow e$ by (3), as desired.

THEOREM 17. Let $S_{1}$ be a semigroup with property (3), and $S_{2}$ another semigroup. Then every continuous isomorphism of $S_{1}$ onto $S_{2}$ is open.

Proof. By Lemma 3 it is sufficient to prove that the mapping $f$ of $S_{1}$ onto $S_{2}$ is open at the identity. Let then $f\left(x_{n}\right)=y_{n} \rightarrow e_{2}=f\left(e_{1}\right)$. We wish to prove that $x_{n} \rightarrow e_{1}$. Let $\epsilon_{i}$ be a sequence of positive real numbers such that $\epsilon_{i} \rightarrow 0$. Since $S_{1}$ is separable it may be covered by countably many $\epsilon_{1}$-spheres. Their images cover $S_{2}$ which, since it is complete, is of the second category. Therefore, one of these $\epsilon_{1}$-spheres, say $T_{1}$, is such that $f\left(T_{1}\right)$ is of the second category. Since $T_{1}$ is a Borel set, $f\left(T_{1}\right)$ is a Suslin set, and, therefore, a Baire set (see [3, p. 249]). Thus there exists a nonempty open set $L_{1}$ in $S_{2}$ such that $L_{1} \cap C f\left(T_{1}\right)$ is of the first category where $C$ denotes the complement; and there exists a nonempty open set $L_{11}$ in $L_{1}$ such that $f\left(T_{1}\right)$ is of the second category at each point of $L_{11}$, while $L_{11} \cap C f\left(T_{1}\right)$ is of the first category. Consider now any $y$ in $L_{11}$. Owing to the continuity of multiplication in $S_{2}$, there exist open sets $L_{12}, L_{13}$ such that $y$ is in $L_{12}$ which in turn is a subset of $L_{11}, e_{2}$ is in $L_{13}$, and $L_{12} L_{13}$ is a subset of $L_{11}$. Since $y_{n} \rightarrow e_{2}$, there exists a natural number $n_{1}$ such that, for $n \geqq n_{1}, y_{n}$ is in $L_{13}$, or $y_{n} L_{12}$ is a subset of $L_{11}$. Note that $f\left(T_{1}\right)$ is of the second cate- 
gory at each point of $L_{12}$ and that $L_{12} \cap C f\left(T_{1}\right)$ is of the first category. Consider now $L_{1 n}=y_{n}\left(L_{12} \cap f\left(T_{1}\right)\right)$ which is of the second category by our above arguments and by Lemma 4 . The equation

$$
L_{1 n}=\left(L_{1 n} \cap f\left(T_{1}\right)\right) \cup\left(L_{1 n} \cap C f\left(T_{1}\right)\right)
$$

shows that $L_{1 n} \cap f\left(T_{1}\right)$ is of the second category, hence not empty, because $L_{1 n}$ is a subset of $y_{n} L_{12}$ which in turn is a subset of $L_{11}$, and $L_{11} \cap C f\left(T_{1}\right)$ and hence $L_{1 n} \cap C f\left(T_{1}\right)$ are of the first category. Select now $y_{1, n}$ in $L_{1 n} \cap f\left(T_{1}\right) . y_{1, n}$ is in $f\left(T_{1}\right)$ or $y_{1, n}=f\left(x_{1, n}\right)$ for some $x_{1, n}$ in $T_{1}$; but $y_{1, n}$ is also in $L_{1 n}$ which is a subset of $y_{n} f\left(T_{1}\right)$, or $y_{1, n}=y_{n} f\left(x_{2, n}\right)$ for some $x_{2, n}$ in $T_{1}, x_{1, n}=x_{n} x_{2, n}$ for $n \geqq n_{1}$.

Assume now that we have a sequence of open spheres in $S_{1}: T_{1}$ $\supset T_{2} \supset \ldots \supset T_{m}, T_{i}$ of radius less than or equal to $\epsilon_{i}$, such that $f\left(T_{i}\right)$ is of the second category; a sequence of natural numbers $0<n_{1}$ $<n_{2}<\cdots<n_{m}$; sequences $x_{2, k}, x_{1, k}=x_{k} x_{2, k}$ for $n_{1} \leqq k<n_{m}$ where, if $n_{i} \leqq k<n_{i+1}, x_{2, k}$ and $x_{1, k}$ are in $T_{i}$. Assume further that for $k \geqq n_{m}$, there exist sequences $x_{2, k}^{\prime}, x_{1, k}^{\prime}=x_{k} x_{2, k}^{\prime}$ such that $x_{2, k}^{\prime}$ and $x_{1, k}^{\prime}$ are in $T_{m}$. We now cover $T_{m}$ by a countable number of open spheres of radii less than or equal $\epsilon_{m+1}$ each of which is contained in $T_{m}$. Since $f\left(T_{m}\right)$ is of the second category, one of these spheres, say $T_{m+1}$, will have an image $f\left(T_{m+1}\right)$ of the second category. Just as in the first part of the proof, we conclude that there exists a nonempty open set $L_{m+1,1}$ contained in $S_{2}$ such that $f\left(T_{m+1}\right)$ is of the second category at each point of $L_{m+1,1}$ while $L_{m+1,1} \cap C f\left(T_{m+1}\right)$ is of the first categcry; that there exists a natural number $n_{m+1}>n_{m}$ and a nonempty open set $L_{m+1,2}$ contained in $L_{m+1,1}$ such that, for $n \geqq n_{m+1}, y_{n} L_{m+1,2}$ is contained in $L_{m+1,1}$; and finally that, for $n \geqq n_{m+1}$, there exist sequences $x_{2, n}^{\prime}, x_{1, n}^{\prime}$ $=x_{n} x_{2, n}$ such that $x_{2, n}^{\prime}$ and $x_{1, n}^{\prime}$ are in $T_{m+1}$.

We now define $x_{2, k}$ and $x_{1, k}=x_{k} x_{2, k}$ for $n_{m} \leqq k<n_{m+1}$ to be equal to $x_{2, k}^{\prime}$ and $x_{1, k}^{\prime}$ respectively. This completes our induction. If we set $x_{2, k}=e_{1}$ for $0<k<n_{1}$, and $x_{1, k}=x_{k} x_{2, k}$, we have defined sequences $n_{i}, x_{2, n}, x_{1, n}=x_{n} x_{2, n}$ for all $n$, such that $0<n_{1}<\cdots$ and, if $n_{i} \leqq n$ $<n_{i+1}, x_{2, n}$ and $x_{1, n}$ are in $T_{i}$ and, therefore, are Cauchy sequences. Owing to the completeness of $S_{1}$ there exists $x_{2}$ such that $x_{2, n} \rightarrow x_{2}$ and also $x_{1, n} \rightarrow x_{2}$, since these two Cauchy sequences are concurrent. Our hypotheses and Lemma 5 now imply that $x_{n} \rightarrow e_{1}$ since we have $x_{n} x_{2, n} \rightarrow x_{2}, x_{2, n} \rightarrow x_{2}$. This concludes our proof.

Note that if $S_{1}$ has the property

(4) there exists a neighborhood of the identity all of whose elements have inverses, and division where possible is continuous, then condition (3) is satisfied. Note also that if the metric of $S_{1}$ is invariant, (3) is true. (The invariance of the metric of $S_{1}$ would ma- 
terially simplify the proof of the above theorem.)

The theorem just proved is a generalization of a theorem of Banach, cf. [1, Theorem 8; 4, Theorem 13].

It is clear that the foregoing theorem will be of use in resolving questions of embeddability of semigroups satisfying the hypotheses for $S_{1}$ if the following are satisfied:

$Q(S)$ is complete (that it is metric is clear); $S^{*}$ is closed in $Q(S)$; $A S^{\#}$ is open in $S^{\#}$ for all $A$ in $S^{\#}$. This last condition implies that if $U^{\sharp}$ is open in $S^{\sharp}$, then so is $A U^{*}$ for all $A$ in $S^{*}$, for $U^{*}=S^{\sharp} \cap U$ for some open subset $U$ of $Q\left(S_{1}\right)$, and $A U^{\sharp}=A\left(S^{\sharp} \cap U\right)=A S^{\sharp} \cap A U$ which is open since $Q\left(S_{1}\right)$ is a group (see Theorem 2).

LEMMA 6. $S^{\sharp}$ is closed in $Q(S)$ if and only if bS is closed in $S$ for all $b$ in $S$.

Proof. We note that $b S$ is closed if and only if $a_{n} \rightarrow a, b \mid a_{n}$ imply $b \mid a$ (where $x \mid y$ means that there exists $z$ such that $y=z x$ )-in other words that the relation | is topological in character. We first note that $b \mid a$ is equivalent to $a$ 's belonging to $b S$. Let now $b S$ be closed, $a_{n} \rightarrow a, b \mid a_{n}$, that is, $a_{n}$ in $b S$. Then $a$ is in $b S$ and $b \mid a$. Let finally $a_{n} \rightarrow a, b \mid a_{n}$ imply that $b \mid a$ and consider $b S$. Let $a_{n} \rightarrow a$ where $a_{n}$ is in $b S$; we wish to show that $a$ is also in $b S$. But $a_{n}$ in $b S$ means that $b \mid a_{n}$; hence $b \mid a, a$ is in $b S$ as desired. Let now $b S$ be closed; we wish to show that $S^{\sharp}$ is closed, that is, if $A_{n}=\phi\left(a_{n}, e\right) \rightarrow A=\phi(a, b)$, then $A$ is in $S^{*}$, that is, $b \mid a$. But $\phi\left(a_{n}, e\right) \rightarrow \phi(a, b)$ implies the existence of $a_{n}^{\prime}, b_{n}^{\prime}$ such that $a_{n}^{\prime} \rightarrow a, b_{n}^{\prime} \rightarrow b, a_{n}^{\prime}=a_{n} b_{n}^{\prime} ; b_{n}^{\prime}=b e_{n}$ (where $e_{n} \rightarrow e$ ), $a_{n}^{\prime}=a_{n} b e_{n}$, or $b \mid a_{n}^{\prime}$; hence $b \mid a$ and $S^{\prime}$ is closed. Lastly, let $S^{\prime}$ be closed. We wish to prove that $b \mid a_{n}, a_{n} \rightarrow a$ imply $b \mid a$ which, by the first part of the present proof, is equivalent to showing that $b S$ is closed. But $b \mid a_{n}$ means that there exists $a_{n}^{\prime}$ such that $a_{n}=a_{n}^{\prime} b$. Thus we have $\phi\left(a_{n}, b\right) \rightarrow \phi(a, b)$; but $\phi\left(a_{n}, b\right)=\phi\left(a_{n}^{\prime}, e\right)$; therefore, $\phi(a, b)$ is in $S^{f}$ as desired.

Note that the fact that $b S$ is closed for $b$ in $S$ implies that $b F$ is closed for all closed subsets $F$ of $S$ and all $b$ in $S: b f_{n} \rightarrow x$ implies that $b \mid x$ or that $x$ is in $b S$, or that $x=b s$; hence we have $b f_{n} \rightarrow b s$ or $b f_{n}=b s e_{n}$ (where $e_{n} \rightarrow e$ ), $f_{n}=s e_{n} \rightarrow s$ in $F, x=b s$ in $b S, b F$ is closed.

Lemma 7. Condition (4) implies that $b S$ is closed for all $b$ in $S$.

Proof. Observe that $b \mid a_{n}, a_{n} \rightarrow a$ imply that $a_{n}=a_{n}^{\prime} b$ for some $a_{n}^{\prime}$, and $a_{n}=a e_{n}$ for $e_{n} \rightarrow e$; therefore, $a e_{n}=a_{n}^{\prime} b$, for $n$ sufficiently large, $a=a_{n}^{\prime} b e_{n}^{-1}$ and $b \mid a$.

We mention two further conditions insuring that $b S$ be closed for all $b$ in $S:$ (1) $b S$ is closed if and only if $b s_{n} \rightarrow x$ implies that there 
exists $s$ such that $s_{n} \rightarrow s$; (2) if the metric of $S$ is invariant, or (which is implied by it) if the mapping $b S \rightarrow S$ is uniformly continuous, then $b S$ is closed for all $b$ in $S$.

Lemma 8. Condition (4) implies that $A S^{*}$ is open in $S^{f}$ for all $A$ in $S^{*}$.

Proof. Note first of all that by an earlier remark this implies that if $U^{\sharp}$ is open in $S^{f}$ and if $A$ is an element of $S^{\sharp}$, then $A U^{\sharp}$ is open in $S^{t}$. Let $A_{n}=\phi\left(a_{n}, e\right) \rightarrow A_{0} B_{0}=\phi\left(a_{0} b_{0}, e\right)$. We wish to prove that $A_{n}=A_{0} A_{n}^{\prime}$ for $A_{n}^{\prime}$ in $S^{\prime}$ and for $n$ large enough. Our hypothesis implies the existence of $a_{n}^{\prime}, b_{n}^{\prime}$ such that $a_{n}^{\prime} \rightarrow a_{0} b_{0}, b_{n}^{\prime} \rightarrow e, a_{n}^{\prime}=a_{n} b_{n}^{\prime}$; but $\left(b_{n}^{\prime}\right)^{-1}$ exists for $n$ large enough; hence $a_{n}^{\prime}\left(b_{n}^{\prime}\right)^{-1}=a_{n}$ and $a_{n}^{\prime}$ $=a_{0} b_{0} e_{n} \quad\left(\right.$ where $\left.e_{n} \rightarrow e\right)$ or $a_{0} b_{0} e_{n}\left(b_{n}^{\prime}\right)^{-1}=a_{n}=a_{0} a_{1, n}$, where $a_{1, n}$ $=b_{0} e_{n}\left(b_{n}^{\prime}\right)^{-1}, A_{n}=\phi\left(a_{n}, e\right)=\phi\left(a_{0} a_{1 n}, e\right)=A_{0} A_{n}^{\prime}$ where $A_{n}^{\prime}=\phi\left(a_{1, n}, e\right)$, as desired. A similar proof shows that (4) also implies that $S^{f}$ is open in $Q(S)$.

These lemmas establish the truth of the following corollary to Theorem 17:

COROLLARY. If $S$ is a locally compact semigroup satisfying condition (4), then $S$ is embeddable in a locally compact group.

Proof. We note that $Q(S)$ is the continuous open image of the locally compact separable metric semigroup $S \times S$; hence it itself is locally compact and separable metric; it is complete because it is a topological group which is locally compact.

The hypotheses of the preceding corollary are not strong enough to make $S$ a group; a counterexample is the multiplicative semigroup of the nonzero $p$-adic integers; nor does condition (4) imply condition (2) -witness the multiplicative semigroup of $p$-adic numbers of absolute value greater than or equal to 2, together with 1 (which is then an isolated point).

\section{BIBLIOGRAPHY}

1. S. Banach, Metrische Gruppen, Studia Mathematica vol. 3 (1931) pp. 101-113.

2. N. Bourbaki, Elements de Mathematique, (a) Book III, chap. I, Actualités Scientifiques et Industrielles, no. 858; (b) Book III, chap. III, Actualités Scientifiques et Industrielles, no. 916.

3. C. Kuratowski, Topologie I, Warsaw-Lw6́w, 1933.

4. L. Pontrjagin, Topological groups, Princeton, 1939.

5. J. W. Tukey, Convergence and uniformity in topology, Princeton, 1940.

UNIVERSITY OF MiNNESOTA 